\title{
Mycobacterium tuberculosis cell wall released fragments by the action of the human lung mucosa modulate macrophages to control infection in an IL-10-dependent manner
}

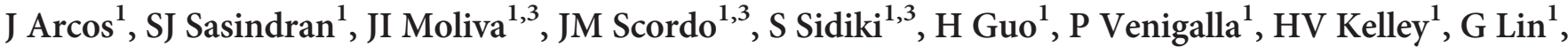 \\ L Diangelo $^{1}$, SN Silwani ${ }^{1}$, J Zhang ${ }^{1,2}$, J Turner ${ }^{1,2}$ and JB Torrelles ${ }^{1,2}$
}

Mycobacterium tuberculosis (M.tb), the causative agent of tuberculosis, is a major public health challenge facing the world. During infection, M.tb is deposited in the lung alveolar space where it comes in contact with the lung mucosa, known as alveolar lining fluid (ALF), an environment that $M$.tb encounters at different stages of the infection and disease. ALF is abundant in homeostatic and antimicrobial hydrolytic enzymes, also known as hydrolases. Here we demonstrate that ALF hydrolases, at their physiological concentrations and upon contact with M.tb, release M.tb cell envelope fragments into the milieu. These released fragments are bioactive, but non-cytotoxic, regulate the function of macrophages, and thus are capable of modulating the immune response contributing to the control of $M$.tb infection by human macrophages. Specifically, macrophages exposed to fragments derived from the exposure of $M$.tb to ALF were able to control the infection primarily by increasing phagosome-lysosome fusion and acidification events. This enhanced control was found to be dependent on fragment-induced interleukin-10 (IL-10) production but also involves the STAT3 signaling pathway in an IL-10-independent manner. Collectively our data indicate that M.tb fragments released upon contact with lung mucosa hydrolases participate in the host immune response to M.tb infection through innate immune modulation.

\section{INTRODUCTION}

Mycobacterium tuberculosis (M.tb) infection is a major public health concern in the world, causing 1.5 million deaths per year. ${ }^{1}$ Although current efforts are directed at the development of new therapies and vaccines, there is still a lack of understanding for how the environment that $M$.tb encounters during its life cycle within the host can influence disease dynamics. ${ }^{2}$ During airborne transmission, $M . t b$ is deposited in the alveolar spaces of the lung where it is exposed to different environments. First, $M$.tb encounters the human lung alveolar space, where M.tb is modified by alveolar lining fluid (ALF) as we have described. ${ }^{2,3}$ Second, after being exposed to ALF, M.tb will encounter alveolar macrophages (AMs), which serve as an important reservoir for infection. ${ }^{4,5}$ In later stages of the infection, $M . t b$ is found extracellular but contained within the granuloma (a cellular structure generated by the host to contain the infection) ${ }^{5,6}$ Finally, $M . t b$ can escape the granuloma(s) and reside extracellularly within cavities where, under suboptimal drug therapy, it can become drug-resistant. ${ }^{7}$

We have recently shown that human lung ALF contains a series of homeostatic and antimicrobial enzymes (called hydrolases) ${ }^{8,9}$ that alter the M.tb cell envelope resulting in modifications of the M.tb cell surface. ${ }^{3}$ The most active enzymes present in healthy human ALF that we have shown to alter the cell wall of M.tb are alkaline (AlkP) and acid (AcP) phosphatases, and a nonspecific esterase (Est). ${ }^{3}$ Our published

${ }^{1}$ Department of Microbial Infection and Immunity, Columbus, Ohio, USA and ${ }^{2}$ Center for Microbial Interface Biology, The Ohio State University, Columbus, Ohio, USA. Correspondence: JB Torrelles (jordi.torrelles@osumc.edu)

${ }^{3}$ These authors contributed equally to this work

Received 18 November 2015; accepted 31 October 2016; published online 21 December 2016. doi:10.1038/mi.2016.115 
studies demonstrate that even a short-term exposure to ALF hydrolases ( $15 \mathrm{~min}$, a plausible time before $M . t b$ contacts a host cell) results in a significant reduction of trehalose dimycolate (TDM) and mannose-capped lipoarabinomannan (ManLAM) on the M.tb cell envelope surface. ${ }^{3}$ TDM and ManLAM are known M.tb virulence factors involved in $M . t b$ intracellular survival. ${ }^{10,11}$ Importantly, these hydrolase-derived M.tb cell envelope surface modifications resulted in a differential recognition by, and altered trafficking and survival within, human phagocytes. ${ }^{3}$

Here we have hypothesized that M.tb fragments released by the action of human ALF hydrolases are capable of directly influencing the host immune response during M.tb infection. Using different human ALFs, our results demonstrate that released M.tb cell envelope fragments induce homotypic cell aggregation, drive human macrophage function towards a type II macrophage phenotype, alter trafficking of phagosomes containing M.tb; and prime macrophages to better control M.tb infection through an increase of phagosome-lysosome fusion events in an interleukin-10 (IL-10)-dependent manner, and through the STAT3 pathway in an IL-10-independent manner. Collectively our data indicate that M.tb fragments released upon contact with human ALF participate in the clearance of infection via innate immune activation. This furthers our understanding of M.tb infection in humans and opens new avenues to enhance mucosal immunity for vaccine development.

\section{RESULTS}

Composition and bioactivity of $M$.tb fragments released by the action of hydrolases present in human ALF

Exposure of M.tb to human ALF alters the M.tb cell envelope. ${ }^{3}$ Here we show that in addition to M.tb cell wall modifications, exposure to ALF releases M.tb cell envelope fragments into the milieu in a consistent and reproducible manner. Using ALF from multiple healthy donors, or relevant in vivo concentrations of AlkP, AcP, and Est (the most abundant enzymes found in human ALF) ${ }^{3}$ alone or in combination (Mix), we show a release of several proteins, peptides and traces of lipoglycans among other M.tb cell wall components (Figure 1a). Importantly, our results demonstrate reproducibility/ homogeneity in the fragments generated (Figure 1b). Our controls, $0.9 \% \mathrm{NaCl}$ and $\beta$-glucosidase [ $\beta$-Glc, a hydrolase detected in human $\operatorname{ALF}^{3}$ that has no activity against the M.tb cell envelope] showed limited fragment release.

Carbohydrate and lipid composition analyses of released fragments showed that these were structurally composed of fatty acids and sugars typically present on the $M$.tb cell envelope (Figure 1c,d). Arabinose (Ara), mannose (Man), and myoInositol (myo-Inos) are sugar constituents of $M . t b$ virulence factors like ManLAM, LM, and PIMs (reviewed in ref. 2). Glucose (Glc) is found in the M.tb cell envelope as trehalose constituting the polar groups of M.tb virulence factors such as sulfolipid-1 and TDM, ${ }^{2}$ among others. The action of ALF hydrolases did not impair bacterial viability as assessed by the ability of M.tb to grow on agar plates (data not shown).
To address the capacity of $M . t b$ cell envelope released fragments to stimulate innate immune cells, human macrophage monolayers (at $2.5 \times 10^{5}$ ) were exposed to fragments corresponding to $5 \times 10^{6}$ bacteria (multiplicity of exposure (MOE) of 20:1) at different time points. Clustering of macrophage monolayers exposed to ALF-M.tb cell envelope fragments was observed as early as $24 \mathrm{~h}$ post exposure (Figure 2a). Controls $(0.9 \% \mathrm{NaCl}$, fragments obtained from incubating $M . t b$ with $0.9 \% \mathrm{NaCl}$ (Figure 2a) or physiological concentrations of human $\mathrm{ALF}^{3}$ ) did not induce macrophage aggregation.

We assessed whether released fragments were able to stimulate cytokine release by macrophages exposed to different MOEs of fragments (5:1, 10:1, 20:1, and 40:1). Our results show that for all MOEs studied, released fragments were capable of inducing the secretion of significant amounts of tumor-necrosis factor (TNF; Figure 2b). Using the same MOEs after $2 \mathrm{~h}, 12 \mathrm{~h}$, and $24 \mathrm{~h}$ exposure, we also show that fragments did not induce cell death (apoptosis and/or necrosis; data not shown).

On the basis of our cytotoxicity and TNF results, we further assessed the production of other cytokines and chemokines implicated in M.tb pathogenesis using the MOE of 20:1. Results show that all $M . t b$ cell envelope fragments induced significant secretion of TNF, IL-6, IL-10, granulocyte-colony stimulating factor (G-CSF), and CCL-5 (Figure 2c). Secretion of IL-12p40, IL-23p19, IL-27p28, IL-1 $\beta$, TGF $\beta$, and CCL-2 were not detected or secretion was not significantly different when compared with controls (data not shown).

We established that the absence of IL-12p40 directly correlated with the ability of fragments to induce IL-10 secretion as neutralization of IL-10 led to a significant increase of IL-12p40 production in response to all fragments tested (Figure 2d). Although there is an established correlation amongst IL-12 family of cytokines and IL-12p 40 production, ${ }^{12}$ the levels of IL-12p40 induced by the fragments before or after IL-10 neutralization did not result in any alteration in the production of IL-23 (not shown) or IL-27 (Figure 2d).

As fragments generate high levels of TNF, IL-6, and IL-10, we further assessed whether production of these cytokines was via Toll-like receptor (TLR) signaling. WT, TLR2-KO, or TLR4$\mathrm{KO}$ mouse cell lines were exposed to M.tb fragments at MOE 20:1. Our results indicate that TNF and IL- 6 induced by fragments were TLR2 dependent. However, IL-10 was independent of both TLR2 and TLR4 (Supplementary Figure S1 online).

Using flow cytometry, we further determined that macrophages exposed to ALF-M.tb released fragments upregulate surface expression of complement receptor 4 (CR4) and the mannose receptor (MR); but not TLR2 (Figure 3a), TLR4, or CR3 (the latter not shown). In addition, levels of CD86 on the macrophage surface did not change suggesting that released fragments may not alter Ag presentation (Figure 3a). This was confirmed by assessing messenger RNA (mRNA) levels of CD86 and HLA-DR (Figure 3b, white vs. black bars in the presence of the isotype). Moreover, for ALF-M.tb-released fragments, the lack of macrophage activation was independent 
a

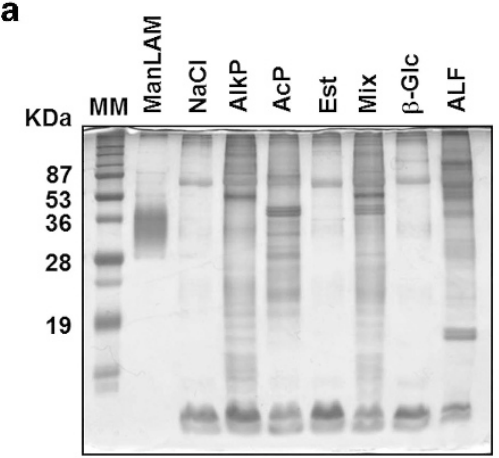

c

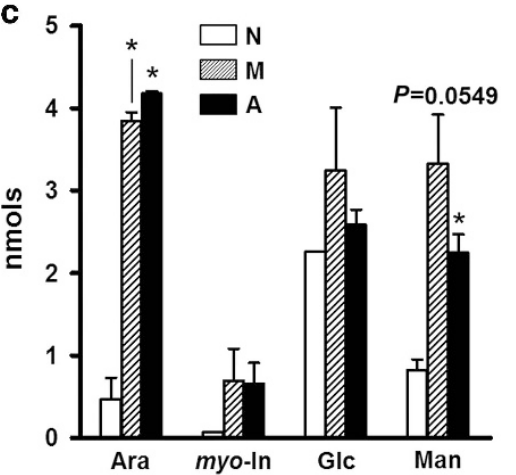

b

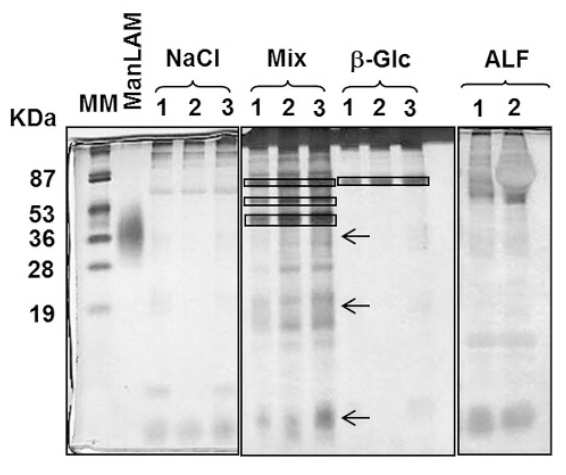

d

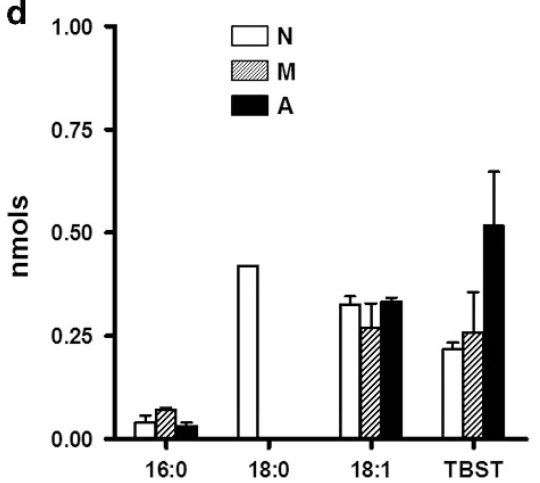

Figure 1 Analysis of ALF exposed-M.tb released fragments. Single-cell suspensions of $M$.tb were exposed to $0.9 \% \mathrm{NaCl}$ (control), human ALF, single hydrolases or to a mixture (Mix) of the most active hydrolases [AlkP $+\mathrm{AcP}+\mathrm{Est}$ ] for $12 \mathrm{~h}, 37^{\circ} \mathrm{C}, 5 \% \mathrm{CO}_{2}$. Supernatants containing released fragments were analyzed. (a, b) A representative SDS-PAGE analysis of released fragments followed by a periodic acid silver stain. (a) M.tb released fragments from $5 \times 10^{6}$ M.tb exposed to different ALF hydrolases and human ALF. AlkP and AcP indicate alkaline and acid phosphatases; Est indicates nonspecific esterase; $\beta$-Glc indicates $\beta$-glucosidase. (b) Released fragments from three independent experiments (1-3) showing reproducibility. Arrows indicate the locations of ManLAM, LM and PIMs on the gel, from high to low molecular mass, respectively, and boxes indicate the location of the hydrolase. (c, d) Released fragments (normalized by bacterial number and volume) were chemically hydrolyzed and their sugar and fatty acid composition determined by mass spectrometry. (c) Neutral sugar analyses $(n=3$, in duplicate) showing that fragments are composed of myo-inositol (myo-In), mannose (Man), glucose (Glc), and arabinose (Ara). (d) Fatty acid analyses of methyl ester derivatives show fragments containing fatty acids ( $n=2$, duplicate). TBST, tuberculostearic acid. Student's $t$-test, ${ }^{\star} P<0.05$. N: fragments generated by M.tb exposure to NaCl; M: fragments generated by $M$.tb exposure to the mixture of hydrolases; A: fragments generated by M.tb exposure to ALF. For each ' $n$ ' value, ALFs were obtained from different human donors. ALF, alveolar lining fluid.

of IL-10 levels, as neutralizing IL-10 did not change the overall macrophage activation status (Figure $\mathbf{3 b}$, comparing black bars).

\section{Effects of $M$.tb-released fragments on the association with and intracellular growth of virulent M.tb within macrophages}

Previously, we demonstrated that ALF-induced alterations of the $M . t b$ cell envelope drive the macrophage to better control M.tb infection. ${ }^{3}$ Here we evaluated whether the presence of fragments further altered the capacity of the macrophage to control M.tb infection. We elected to add M.tb and fragments simultaneously as we envision this to be physiologically relevant in vivo. In their presence (+ Frag), macrophages had a significant increased capacity to control ALF-exposed M.tb (ALF-M.tb) intracellular growth (Figure 4a,b). The additive effects of the released fragments during M.tb infection were evident as early as $72 \mathrm{~h}$ post infection.

Because fragments drive the secretion of several cytokines and growth factors implicated in controlling M.tb growth within macrophages, ${ }^{5}$ we next assessed whether blocking them altered the capacity of macrophages to control M.tb infection. Our results show that control of ALF-M.tb infection is TNF, IL-6, and G-CSF independent, as their neutralization did not alter the capacity of macrophages to control better the infection in the presence of fragments (Figure 4c). Instead, and in contrast to published studies, ${ }^{13}$ we determined that fragmentdriven control of ALF-M.tb infection is dependent on IL-10, as we observed a reversal of the enhanced control of $M$. $t b$ infection when IL-10 was neutralized by Ab (Figure 4c). This was not observed in the control $(0.9 \% \mathrm{NaCl}$ exposed M.tb).

Importantly, although fragments increased the surface expression of CR4 and the MR (both phagocytic receptors to which $M . t b$ binds) on the macrophage cell surface, this did not lead to altered recognition of M.tb by macrophages in the presence of fragments (Supplementary Figure S2) as we have previously shown for ALF-exposed M.tb, ${ }^{3}$ and thus, the enhanced control of $M$.tb intracellular growth by macrophages in the presence of the fragments is not dependent on differences in $M$.tb association with macrophages.

Considering that ALF/hydrolase-exposed M.tb bacilli in the presence of their released fragments demonstrated reduced 


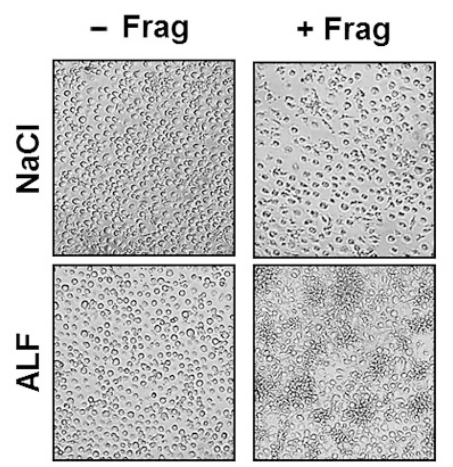

b

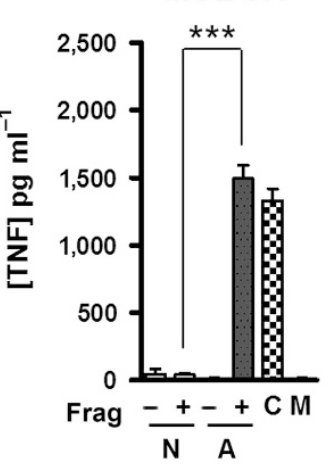

MOE 10:1

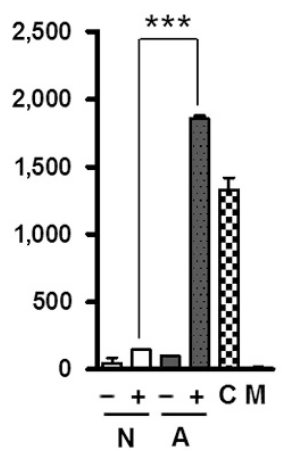

MOE 20:1

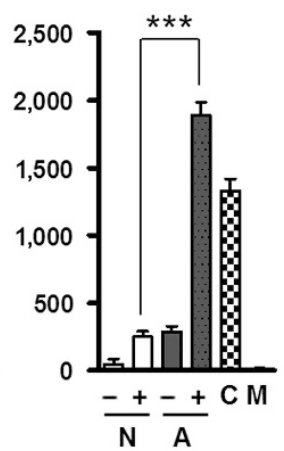

MOE 40:1

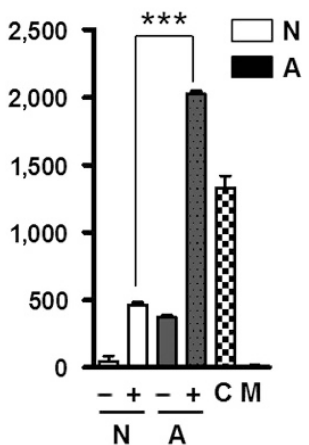

C

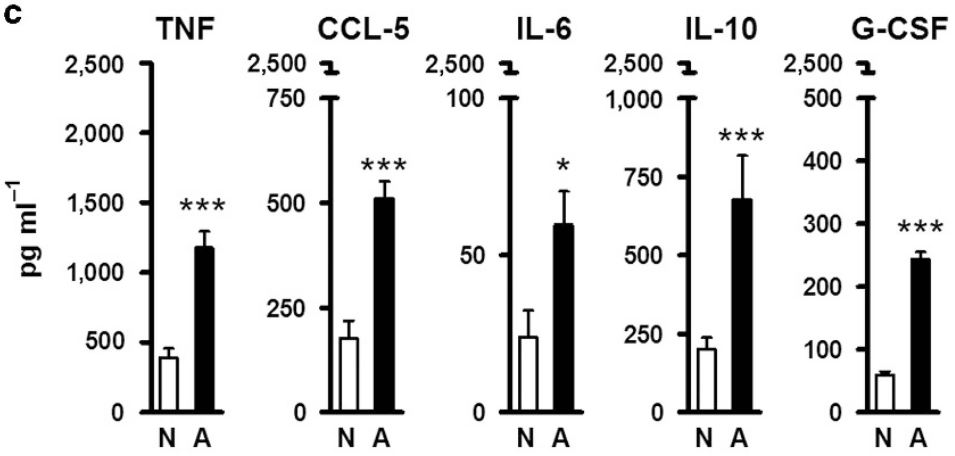

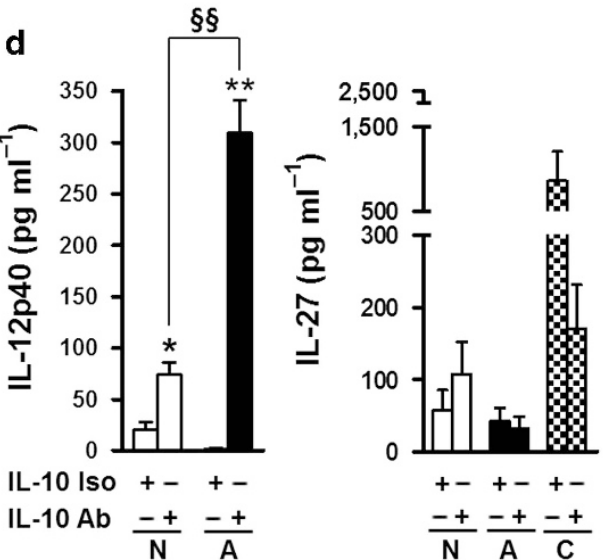

Figure 2 Cytokine and chemokine production induced by macrophages exposed to M.tb cell wall fragments. (a) Macrophages exposed to M.tb cell wall fragments trigger cell aggregation. Human macrophage monolayers were exposed to fragments $(+\mathrm{F})$ or controls $(-\mathrm{F})$ for $24 \mathrm{~h}$ at $37^{\circ} \mathrm{C}, 5 \% \mathrm{CO}_{2}$. Monolayers exposed to human ALF developed cell aggregations. Aggregations were observed as early as $12 \mathrm{~h}$ (data not shown) and increased in number and size through $240 \mathrm{~h}$ until the monolayer lost integrity. Fragments obtained upon $\mathrm{M}$.tb exposure to $0.9 \% \mathrm{NaCl}$ or exposure to $\beta$-Glc (not shown) served as controls. (b) TNF production by human macrophages exposed to fragments at different multiplicity of exposure (MOE). Human macrophages were exposed to fragments $(+\mathrm{F})$ or controls $(-\mathrm{F})$ at different MOE. A representative $(\mathrm{M} \pm \mathrm{s}$.d.) experiment in triplicate of $n=3$ is shown. Student's $t$-test was used to compare fragments generated by M.tb exposure to ALF with fragments generated by M.tb exposure to NaCl, ${ }^{* * *} P<0.0005$. C: LPS (positive control), M: Media (background). (c) Human macrophage monolayers were exposed to M.tb cell wall fragments at an $\mathrm{MOE}^{\circ}$ of $20: 1$ for $12 \mathrm{~h}, 37^{\circ} \mathrm{C}, 5 \% \mathrm{CO}_{2}$, and cytokine and chemokine secretion was measured from cell supernatants by ELISA; between $n=4$ and 9 experiments were performed depending of the measured immune component in triplicate. (d) IL-12p40 and IL-27 secretion in the absence [( -$)$ isotype control] or presence (+) of anti-IL-10 neutralizing antibody $(n=3)$. In (c/d), shown $M \pm$ s.e.m., Student's $t$-test was used to compare the immune response induced by fragments generated by M.tb exposure to ALF with fragments generated by M.tb exposure to $\mathrm{NaCl}$ in the absence $\left({ }^{*} P<0.05 ;{ }^{* *} P<0.005 ;{ }^{* * *} P<0.0005\right)$ or in the presence of antiIL-10 Ab $\left({ }^{\S} P<0.005\right)$. NaCl-Frag [N]; ALF-Frag [A]. For each ' $n$ ' value, both ALF and macrophages were obtained from different human donors. ALF, alveolar lining fluid; IL, interleukin; TNF, tumor-necrosis factor.

early intracellular growth in macrophages, we next evaluated whether phagocytosis of exposed bacilli in the presence of fragments resulted in altered intracellular trafficking. We incubated human macrophages with control M.tb $(0.9 \% \mathrm{NaCl}$ exposed M.tb) or with M.tb exposed to human ALF in the presence or absence of fragments, and assessed phagosomelysosome (P-L) fusion events for up to $2 \mathrm{~h}$. Our results show that uptake of ALF-exposed M.tb in the presence of released fragments results in an increase in the overall level of $\mathrm{P}-\mathrm{L}$ fusion events ( $\sim 25 \%$, CD 63$)$ and compartment acidification $(\sim 15 \%$, Lyso-T; Figure 5a). Importantly, we confirmed that the increased P-L fusion observed in response to ALF-exposed $M . t b$ and fragments combined was driven by the presence of IL-10 (Figure 5b). This phenomenon was exclusively observed for ALF-exposed M.tb (black bars), where neutralizing IL-10 results in a decrease in P-L fusion events. In contrast and as published by others, control, NaCl-exposed M.tb (white bars), showed an increase in P-L fusion events when IL-10 was neutralized. In addition, the level of autophagy, an alternate cellular mechanism to control M.tb intracellular growth, was also increased to a small extent ( $\sim 10 \%$, LC-3; Figure 5a). However, of those autophagosomes observed, only 30\% were acidified (Figure 5c). Thus, the small increase in autophagy does not explain the increased M.tb control observed.

Macrophage control of ALF-exposed M.tb in the presence of fragments was partially dependent on IL-10 induced by the fragments. As fragments also induced the production of IL-6 and G-CSF, which together with IL-10 activate the same signaling pathway (STAT3), we further assessed whether the enhanced control of ALF-exposed M.tb in the presence of 

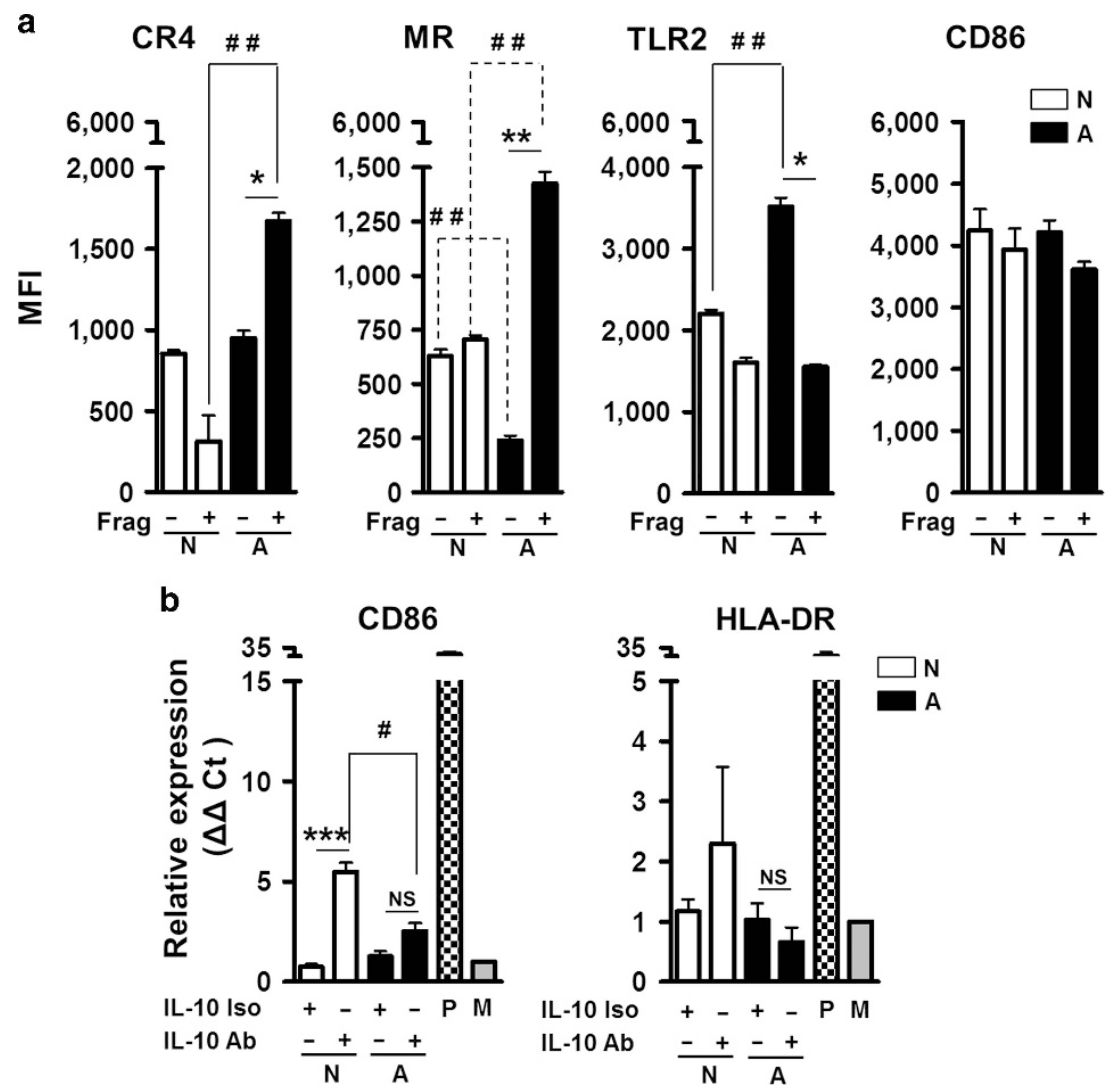

Figure 3 Effects of ALF fragments on macrophage receptors and activation. (a) Macrophages exposed to ALF exposed M.tb $[\mathrm{A}]$ or $0.9 \% \mathrm{NaCl}$ exposed $M . t b[\mathrm{~N}]$ released cell wall fragments induce the surface expression of the MR and CR4, but not TLR2. Differences in the macrophage activation marker CD86 on the macrophage surface were not observed. Experiments were performed $n=3$ and cells were analyzed by flow cytometry. Shown M \pm s.e.m., Student's $t$-test was used to compare the receptor/marker surface expression induced by fragments generated by M.tb exposure to ALF with fragments generated by M.tb exposure to $\mathrm{NaCl}\left({ }^{\star} P<0.05 ;{ }^{* \star} P<0.005\right)$. (b) Expression of CD86 and HLA-DR as measured by RT-PCR $(n=3)$. P: Positive Control; M: Medium. Student's $t$ test was used to compare the mRNA levels induced by fragments generated by M.tb exposure to ALF with fragments generated by M.tb exposure to $\mathrm{NaCl}$ in the presence $\left({ }^{\#} P<0.05 ;{ }^{* * *} P<0.0005\right)$ or in the absence of anti-IL-10 Ab. For each ' $n$ ' value, both ALF and macrophages were obtained from different human donors. ALF, alveolar lining fluid; IL, interleukin.

fragments is dependent on the phosphorylation/activation of STAT3. Results show that ALF-exposed M.tb in the presence of fragments leads to a decrease in pSTAT3 and an increase in NF-кBp65 (Figure 6a,b). These changes were IL-10 independent. Complete inhibition of STAT3 and/or NF- $\mathrm{B}$ further enhanced the control of $M . t b$ by macrophages over that seen for ALF-M.tb in the presence of fragments (Figure 6c).

We also determined whether ALF-exposed M.tb-released fragments could stimulate macrophages to produce reactive oxygen intermediates as another mechanism to explain the limited M.tb growth observed. Macrophages were exposed to fragments and the assembly of the NADPH oxidase complex was assessed by measuring phosphorylation $(\mathrm{P})$ of the cytosolic subunit $\mathrm{p} 40^{p h o x}$. Our results showed no difference in the levels of P-p40 phox induced by ALF- or Mix-fragments when compared with $\mathrm{NaCl}$ fragments (Supplementary Figure S3A). This lack of difference was further confirmed by flow cytometry, directly measuring the formation of superoxide induced by fragments (Supplementary Figure S3B). Inducible nitric oxide synthase (iNOS), which produces the metastable free radical nitric oxide, is also involved in the killing of $M . t b ;{ }^{14}$ however, our results showed that fragments did not induce iNOS (Supplementary Figure S3C). Finally, similar to the results obtained with macrophages exposed to fragments, we determined that ALF-exposed M.tb infection in the presence of fragments do not induce apoptosis/necrosis (data not shown). These results rule out the contribution of macrophage death in the enhanced control of ALF-M.tb infection.

Overall, our results indicate that the enhanced intracellular control of M.tb growth by macrophages in the presence of fragments is independent of the oxidative response, and largely dependent on IL-10-mediated increase in P-L fusion. Downregulation of pSTAT3 and upregulation of NF-кBp65 also contributes to increased control of M.tb growth in an IL-10-independent manner.

\section{DISCUSSION}

The TB field has elucidated and targeted numerous released/ secreted antigens and structural motifs of the $M$.tb cell envelope that are considered critical in triggering host immune responses during M.tb infection. ${ }^{5,15}$ Although these studies are important, there has been little consideration that 

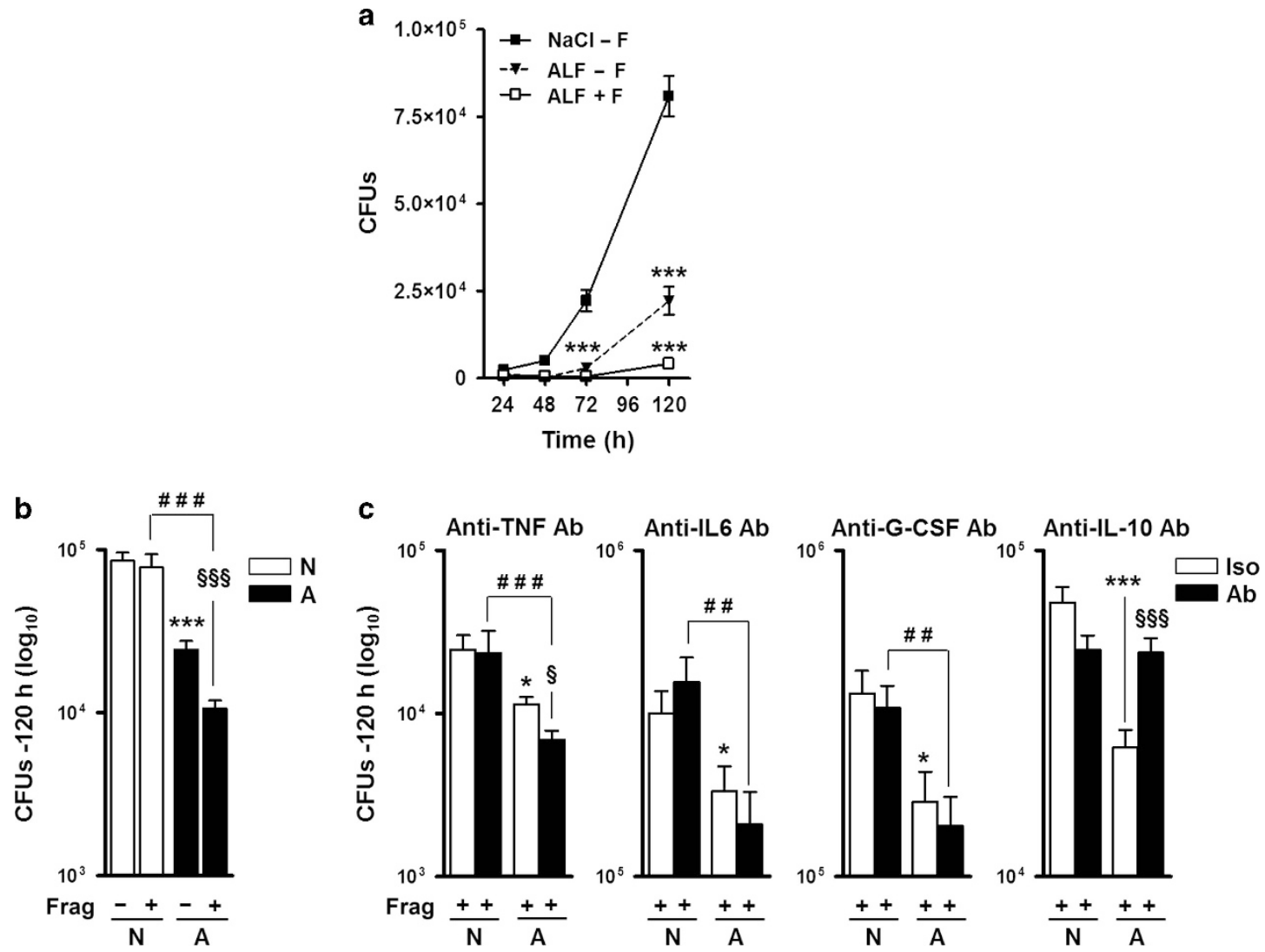

Figure 4 Effects of ALF on M.tb intracellular survival in human macrophages. M.tb bacilli were exposed to $0.9 \% \mathrm{NaCl}$ (control), Mix, or ALF at relevant in vivo concentrations. Macrophages were infected at a MOI 1:1 with exposed M.tb in the absence or presence of fragments and in the absence or presence of anti-TNF, anti-IL-10, anti-IL-6 or anti-G-CSF neutralizing Abs. Intact macrophage monolayers were verified by inverted phase microscopy throughout the infection period. M.tb survival was determined by colony-forming units (CFUs) at the indicated intervals. (a) A representative experiment in triplicate is shown ( $\mathrm{M} \pm$ s.d.), Student $t$ test was used to compare control with vs. without fragments $( \pm \mathrm{F})$, ${ }^{* \star *} P<0.0005$. (b) Overall data at $120 \mathrm{~h}$ from $n=3$ each performed in triplicate (M \pm s.e.m.). (c) Effects of neutralizing TNF, IL-10, IL-6 or G-CSF on macrophages in the presence of fragments during exposed M.tb infection ( $n=3$ in triplicate, $\mathrm{M} \pm$ s.e.m.). Notice that y-axes are different among the graphs shown. One-way ANOVA, Tukey-Posttest was used to compare the effects on the M.tb cell wall ( ${ }^{*}$, white bars), $\pm \mathrm{F}$ (§, white vs. black bars), and M.tb exposed to different conditions in the presence of fragments (\#, black bars), ${ }^{\star} P<0.05$; ${ }^{\star *} P<0.005$; ${ }^{* \star \star} P<0.0005 ;{ }^{\circledR} P<0.05 ;{ }^{\$ s \$} P<0.0005$; ${ }^{\# \#} P<0.005$; ${ }^{\# \# \#} P<0.0005$. NaCl-M.tb + Frag $[\mathrm{N}]$; ALF$M . t b+$ Frag Frag $[A]$. For each ' $n$ ' value, both ALF and macrophages were obtained from different human donors. ALF, alveolar lining fluid; G-CSF, granulocyte-colony stimulating factor; IL, interleukin; TNF, tumor-necrosis factor.

homeostatic enzymes present in human lung ALF can alter the properties of the M.tb cell envelope during infection. In this study, we have extended our previous work ${ }^{3}$ and evaluated the impact of released M.tb fragments by the action of ALF hydrolases on human macrophages in vitro. Our results indicate that ALF-released M.tb fragments are bioactive and thus, capable of influencing macrophage function before they encounter M.tb. This scenario is something that, to our knowledge, has not been previously considered or described. Importantly, although ALF-released M.tb fragments did not alter the ability of macrophages to recognize ALF-exposed $M . t b$, released fragments significantly increased the ability of macrophages to control M.tb (increased phagolysosome fusion/acidification and subsequent decreased growth). Interestingly, the enhanced control of ALF-exposed M.tb by macrophages was not mediated by TNF, IL-6, or G-CSF, but instead was mediated by the presence of IL-10 induced by fragments and the transcription factors STAT3/NF-kB.
In contrast to our findings, IL-10 has previously been shown to block phagolysosome maturation, thus facilitating M.tb intracellular growth. ${ }^{13}$ Our results challenge this concept, as neutralizing IL-10 induced by fragments led to decreased phagolysosomal maturation of ALF-exposed M.tb. Therefore, the IL-10 levels generated by the presence of ALF-exposed M.tb fragments are responsible (directly or indirectly) for orchestrating macrophage-mediated control of ALF-exposed M.tb and intracellular growth in human macrophages in vitro. IL-10 has been shown both in vivo and in vitro to be detrimental in controlling M.tb infection, although these studies were performed without considering the impact of the lung mucosa on the M.tb cell wall. Polymorphisms in the $I L-10$ gene in humans have also been associated with increased susceptibility to TB, and several IL-10-deficient mouse studies have shown a long-term detrimental role of IL-10 in mycobacterial infections. ${ }^{16}$ However, mouse studies have not dissected the very first encounter of $M . t b$ with host cells, where differences may be reflected in our findings. In the present study, we focused on 
a
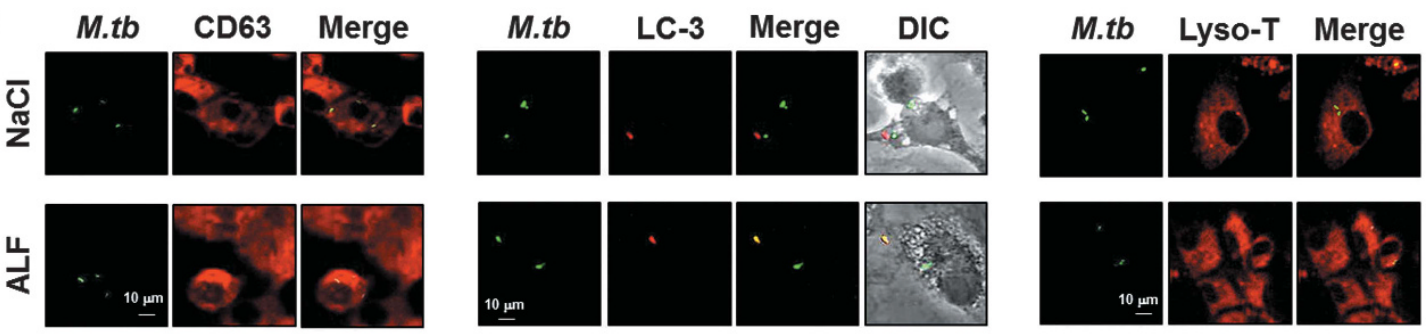

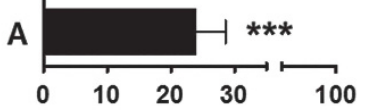

$\%$ Increase vs. $\mathrm{NaCl}+\mathrm{F}$

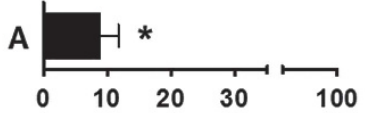

$\%$ Increase vs. $\mathrm{NaCl}+\mathbf{F}$

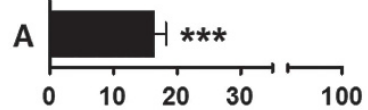

$\%$ Increase vs. $\mathrm{NaCl}+\mathrm{F}$ b

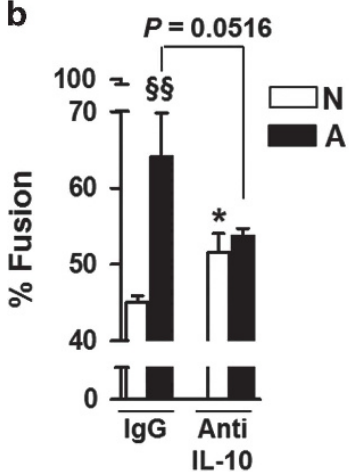

C

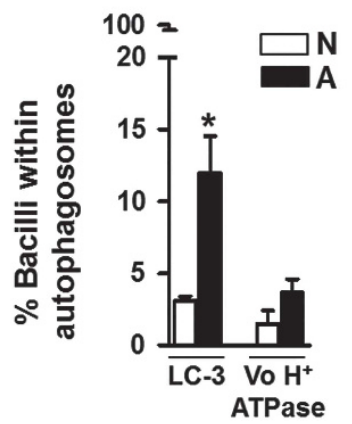

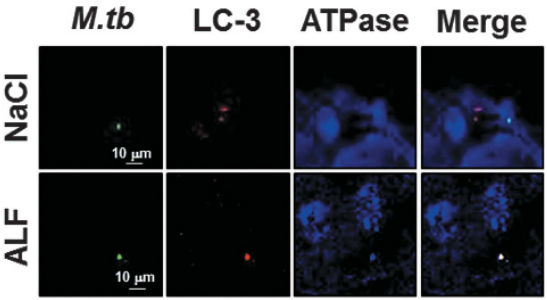

Figure 5 Effects of human ALF on phagolysosome (P-L) fusion in macrophages. Macrophage monolayers on coverslips were incubated with NaCl- or ALF-exposed-GFP-M.tb (multiplicity of infection (MOI) 10:1) in the presence (multiplicity of exposure (MOE) 10:1) or absence of their respective released fragments $( \pm F)$ for $2 \mathrm{~h}$. The cells were washed, fixed, permeabilized, and stained with anti-human CD63, LC-3, $\mathrm{V}_{0} \mathrm{H}^{+}$ATPAse, their respective IgG controls, or with Lyso-Tracker (Lyso-T) (a) Shown are merged images where CD63 (left panel), LC-3 (middle panel), or Lyso-T (right panel) positive compartments are red, GFP-exposed-M.tb bacilli in unfused vesicles are green, and those co-localized are yellow. Phagolysosome (CD63/M.tb), autophagosome (LC-3/M.tb) fusion events, and acidification (Lyso-T/M.tb) were examined and enumerated via confocal microscopy, $n=3-4$ counting $>150$ events per coverslip, in triplicate (original magnification $\times 600$ ). Overall percent increase from $n=3-4$ in triplicate $(M \pm s$.e.m.); Student's $t$-test was used to compare ALF exposed M.tb with $\mathrm{NaCl}$ exposed $M$.tb (control) in the presence of fragments, ${ }^{\star} P<0.05$; ${ }^{\star \star} P<0.005$; ${ }^{* \star \star} P<0.0005$. (b) $\mathrm{P}-\mathrm{L}$ fusion was quantified in the presence of anti-IL-10 neutralizing Ab. Student's $t$-test was used to compare ALF exposed M.tb with NaCl-exposed M.tb (control) in the presence of fragments, ${ }^{\star} P<0.05$ (between white bars), ${ }^{\$ \S} P<0.005$ (between white and black bars under the same condition). (c) Shown are merged images where LC-3 positive compartments are red, $\mathrm{V}_{0} \mathrm{H}^{+}$ATPAse compartments are blue, and those co-localized are yellow. Student's $t$-test was used to compare Mix or ALF exposed M.tb with $\mathrm{NaCl}$-exposed M.tb (control) in the presence of fragments, ${ }^{\star} P<0.05$. For each ' $r$ ' value, both ALF and macrophages were obtained from different human donors. ALF, alveolar lining fluid; IL, interleukin.

determining how alterations that are induced by the human lung mucosa on the M.tb cell wall influence the interaction of macrophages and ALF-M.tb. We demonstrate that macrophages can better control ALF-M.tb infection, and macrophages lose this ability only when IL-10 induced by the fragments is blocked. Adding exogenous IL-10 (mimicking the effects of fragments) to assess whether we could artificially reproduce the effect of IL-10 did not enhance the better control of ALF-M.tb by macrophages (Supplementary Figure S4). This could be explained by the complexities of fragments-M.tbcell interactions, as well as by the timing and location of IL-10IL-10R binding. Thus, IL-10 neutralization is the optimal way to evaluate the role of IL-10 in this instance.

Blocking IL-6 or G-CSF did not change (or increased to a small extent in some cases) the capacity of macrophages to control the infection. This phenomenon was only observed in ALF-exposed M.tb, indicating that ALF-induced changes to the
$M . t b$ cell wall are responsible for the phenotype. In addition, we assessed whether the potential effects of $M$. $t b$-fragments could trigger a change in macrophage phenotype and function before and after uptake of M.tb. Although we determined that fragments could alter the macrophage cell surface expression of several pattern recognition receptors involved in M.tb pathogenesis, ${ }^{15}$ these differences did not alter $M . t b$ association with macrophages (likely as a result of decreased presence of $M$.tb ligands following ALF exposure ${ }^{3}$ ), nor alter expression of important proteins involved in $\mathrm{Ag}$ presentation. In support of our findings that indicate a partial role for IL-10 in the capacity of the macrophage to better control the growth of ALF-M.tb, in vivo studies using $\mathrm{IllO}^{-1-}$ mice highlight different outcomes for a variety of intracellular infections; where in some instances, the absence of IL-10 can promote the propagation of infection. ${ }^{16-19}$ On the basis of our overall data, we reason that the macrophage IL-10-dependent mechanism of M.tb 
a

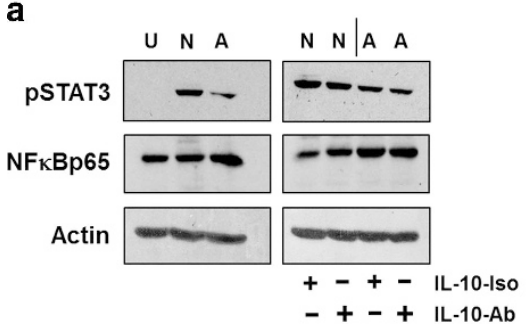

C

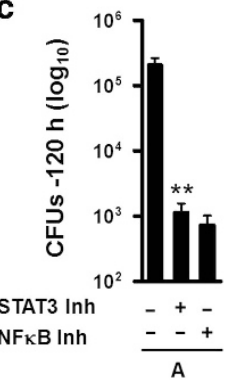

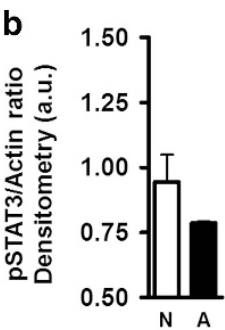
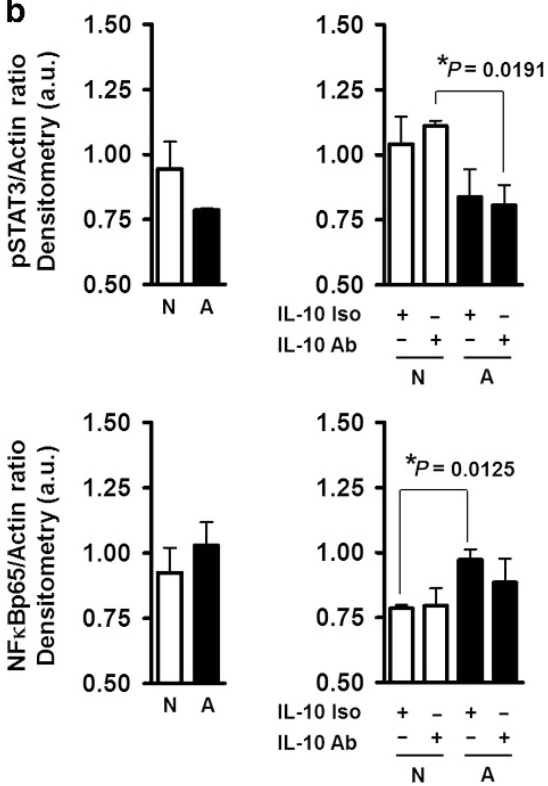

Figure 6 Effects of ALF on macrophage STAT3 and NF- $\mathrm{B} B$ activation. Following infections as described in Figure 4, lysates from ALF-M.tb [A] or NaCl$M . t b[\mathrm{~N}]$ infected macrophages in the presence of their respective fragments were obtained. (a) Representative western blots show that ALF-exposed $M . t b$ in the presence of fragments decreased the activation of STAT3 signaling pathway and increased NF-kB activation. This was not an interleukin-10 (IL-10) dependent mechanism. U: uninfected; $\mathrm{N}$ and A: NaCl- or ALF-exposed M.tb in the presence of fragments, respectively. (b) Densitometry analysis of $n=3$ showing phosphorylated STAT3 and NF-кB vs. Actin ratios. Student's $t$-test was used to compare ALF-exposed M.tb with NaCl-exposed M.tb (control) in the presence of fragments, ${ }^{\$ \$} P<0.005$ (between white and black bars under the same condition). (c) Inhibition of STAT3 and NF- $\mathrm{KB}$ both allowed macrophages a further enhanced control of ALF-M.tb infection in the presence of fragments. Student's $t$-test was used to compare ALF-exposed $M . t b$ infection in the presence of fragments in the absence or presence of inhibitors, ${ }^{\star \star} P<0.005$. For each ' $n$ ' value, both ALF and macrophages were obtained from different human donors. ALF, alveolar lining fluid; a.u., arbitrary unit.

control could be linked to regulation of IL-12 cytokine family members (i.e., IL-12, IL-27). However, although the IL-12 cytokine family can regulate phagolysosome fusion and acidification in human macrophages, ${ }^{20}$ fragments did not induce these cytokines at detectable levels, and only induced IL-12p40 production after IL-10 was neutralized. Low production of IL-27 triggered by the fragments could add to the phenomenon observed, as low levels of IL-27 can generate a pro-inflammatory cytokine storm that can assist in the initial control of the infection. ${ }^{20,21}$

On the basis of our results, we conclude that IL-10 levels may define the initial balance towards $M$.tb intracellular killing or survival within macrophages, where IL-10 (in combination with other fragment induced cytokines) may direct a delicate equilibrium between suppressing and/or activating macrophage intracellular killing mechanisms via STAT3 and/or NF$\kappa \mathrm{B}$ phosphorylation that may determine the establishment of $M . t b$ infection. In this regard, we have determined that macrophages exposed to M.tb-released fragments behave differently, thus indicating a change in macrophage functional capacity. This is in agreement with published studies showing that inhibition of STAT3 enhances phagosome maturation using M.tb $\mathrm{H}_{37} \mathrm{R}_{\mathrm{a}}$ and PMA-differentiated THP-1 cells. ${ }^{13}$

Macrophages have been defined as classically activated, alternatively activated or a type II-activated phenotype. ${ }^{22}$ Classically activated macrophages are induced by microbial products and support a Th1 immune response. Conversely, macrophages become immunosuppressive after being alternatively activated by IL-4. Type II-activated macrophages also act as an anti-inflammatory cell, preferentially inducing a Th2 immune response after their initial activation in a Th1-type environment. ${ }^{22}$ In type-II macrophages, IL-12p40 is reduced and the IL-10 immunomodulatory cytokine is increased. We observed that fragments induced macrophage secretion of IL-10, but also induced secretion of significant amounts of TNF and IL-6 (but not IL-12p40 and CCL-2), thus driving a characteristic cytokine/chemokine profile akin to type IIactivated macrophages. ${ }^{22}$ In this regard, fragments were also able to induce IL-12p40 production but only after IL-10 neutralization, ${ }^{23,24}$ driving a cytokine profile more consistent with classically activated macrophages. ${ }^{22,25}$ Thus, the presence of fragments in the lung may change the macrophage phenotype and function favoring the conversion from an alternative activated to a type II (or IL-10 driven) phenotype. ${ }^{26}$ This change could impact how the macrophage responds during long-term $M . t b$ infection. Further studies are in progress to test this hypothesis.

ALF-released M.tb fragments contain sugar and fatty acids related to cell wall components that we have previously shown to be significantly reduced on the cell envelope surface of ALFexposed M.tb (i.e., ManLAM, TDM). ${ }^{3}$ Although we observed that ALF-released M.tb fragments do not induce macrophage apoptosis/necrosis before or during infection (data not shown), they induce significant secretion of TNF, IL-6, IL-10, G-CSF, 
and the chemoattractant CCL-5 (RANTES) but not IL-12p40, IL-23, IL-27, IL-1 $\beta$, and the inflammatory chemokine CCL-2 (MCP-1). The production of TNF and IL- 6 by these fragments was in part TLR2, but not TLR4, dependent. However, the production of IL-10 was independent of both TLR2 and TLR4. In this regard, the increased expression of the MR observed on the cell surface of macrophages exposed to ALF-M.tb-released fragments could implicate a MR-specific signaling pathway triggering IL-10 production. ${ }^{5}$ The lack of CCL-2 production by fragments rules out the role of this chemokine in the observed inhibition of IL12p40 production. ${ }^{27}$ The induction of CCL-5 (maintained at least for $96 \mathrm{~h}$ after infection in vitro (data not shown)) but not CCL-2 may explain the macrophage aggregation induced by fragments. In this regard, CCL-5 has been shown to directly participate in monocyte/macrophage/T cell activation resulting in greater cell aggregation ${ }^{28}$ and is also significantly expressed in granulomatous tissue, ${ }^{29,30}$ the latter a phenomenon that TNF, IL- $1 \beta$, or IL- 6 cannot induce alone. ${ }^{28}$ The production of TNF, IL-6, and CCL-5 is also observed in macrophage cultures that had been exposed to fragments obtained after exposure of M.tb to ALF for a short time (i.e., $15 \mathrm{~min}$; data not shown).

TNF also downregulates IL-12p40 production, ${ }^{31}$ and thus could also be implicated in a direct negative feedback mechanism, perhaps by regulating IL-12p40/IL-27 pathways to induce anti-mycobacterial activity. However, our data support the paradigm that after M.tb is exposed to ALF, the control of M.tb intracellular growth by macrophages is regulated in a TNF-independent manner. Thus, the high TNF production observed could not explain the enhancement of $\mathrm{P}-\mathrm{L}$ fusion events (between 10 and $20 \%$ increased), a bacterial killing mechanism that can also be enhanced upon macrophage autocrine secretion of TNF. ${ }^{32} \mathrm{P}-\mathrm{L}$ fusion alone accounts for the $\sim 0.9 \log _{10}$ reduction in M.tb intracellular growth in macrophages exposed to released fragments. Thus, increased $\mathrm{P}-\mathrm{L}$ fusion does not solely explain why neutralization of TNF fails to reverse the control of ALF-exposed M.tb growth within macrophages, a phenomenon that is independent of the presence or absence of fragments.

If we look at the overall effect of ALF hydrolases on the M.tb cell wall, our results indicate that the basal environmental state of the human lung has a significant impact on M.tb infection, where ALF hydrolase-derived modifications of the M.tb cell wall plus the effects of the released fragments on host cells allows the human macrophage to better control M.tb growth in vitro (i.e., an $\sim 1.65 \log _{10}$ reduction observed for ALFexposed M.tb plus fragments when compared with $0.9 \% \mathrm{NaCl}$ exposed M.tb). However, exposure to fragments also triggers the formation of autophagosomes, of which only $\sim 30 \%$ were acidified; suggesting that exposure to fragments may also favor the formation of autophagosomes as a potential niche for M.tb intracellular survival in macrophages.

Although fragments induced significant amounts of TNF, this high TNF production did not generate the extracellular release or intracellular production of NOIs and reactive oxygen intermediates, or induce macrophage death by apoptosis. ${ }^{5}$ An explanation could be that the production of IL-10, IL-6, and G-CSF induced by fragments contribute inhibiting the oxidative response. ${ }^{16,33}$

Any differences observed between host cells in the presence or absence of fragments during M.tb infection indicate a direct involvement of the alveolar hydrolase generated fragments on the capacity of alveolar resident cells to control M.tb infection. We observed that the presence of fragments alone did not further influence the macrophage capacity of recognizing and binding $M . t b$, regardless of whether macrophages were preexposed to fragments for short $(30 \mathrm{~min})$ or long $(720 \mathrm{~min})$ periods of time, or at the same time of infection. However, the presence of fragments provided an additive effect on the control of intracellular growth of M.tb within human macrophages. Thus, although released fragments do not impact M.tbmacrophage recognition, they could regulate the function of macrophages by lifting the block to phagosome maturation, which explains the enhancement of P-L fusion events that we observed in the presence of fragments. Phagosome maturation has been related to the presence of pro-inflammatory cytokines activating the macrophage. ${ }^{34}$ Phagosome maturation, however, is suppressed in macrophages capable of producing IL-10. ${ }^{34-36}$ As released fragments induce both pro-inflammatory cytokines (TNF, IL-6) and the immunomodulator IL-10, a balance in their production ratio towards TNF/IL-6 may explain the small but significant increase in phagosome maturation observed in the presence of fragments.

In summary, our studies indicate that pulmonary ALF hydrolases can alter the outcome of infection. A role for ALF hydrolases is plausible. Healthy people are estimated to have approximately 8-12 AMs per alveolar sac and, ${ }^{37,38}$ in this scenario, we believe that $M . t b$ will remain submerged within the ALF hypophase for some time and thus be in contact with ALF hydrolases as well as other ALF components. ${ }^{39}$ We predict that the action of human lung hydrolases on the $M$. $t b$ cell envelope results in the exposure of modified surface molecules ${ }^{3}$ and released cell wall fragments whose impact on the host cell has not yet been appreciated. A link between the level of macrophage activation and M.tb intracellular survival has been suggested in the past; ${ }^{5}$ however, how these events connect with the specific nature of the lung environment, influencing host-pathogen crosstalk and the outcome of M.tb infection, is still unknown. Our current studies will address how human lung hydrolase effects on the M.tb cell wall affect the course of infection in vivo.

\section{METHODS}

Ethics statement. This study was carried out in strict accordance with US Code of Federal and Local (University IRB) Regulations, and Good Clinical Practice as approved by the National Institutes of Health (NIAID/DMID branch).

Chemical reagents, antibodies, and cell lines. All chemical reagents were of the highest grade from Sigma-Aldrich (St. Louis, MO) unless otherwise specified. For specific reagent information, refer to Supplementary Material online. 
Growth conditions of M.tb. GFP-M.tb Erdman (provided by Dr Horwitz, UCLA) strain was grown as we previously described. ${ }^{3}$

Human ALF isolation. ALF was obtained from human bronchoalveolar lavage fluid as we previously described. ${ }^{3}$ See Supplementary Material online for details. ALF (defined in this study as bronchoalveolar lavage fluid $>10 \mathrm{KDa}$ fraction) was frozen at $-80^{\circ} \mathrm{C}$ until use.

Hydrolase and ALF exposure of M.tb and production of M.tbreleased fragments. $M$. $t b$ exposure to human ALF or hydrolases was performed as we have previously described. ${ }^{3}$ Briefly, single-cell suspensions of $M . t b\left(1 \times 10^{8}\right)$ were incubated for $12 \mathrm{~h}, 37^{\circ} \mathrm{C}, 5 \% \mathrm{CO}_{2}$ with $0.9 \% \mathrm{NaCl}$ (control), human $\mathrm{ALF}$ in $0.9 \% \mathrm{NaCl}$ or with a hydrolase mixture (Mix) in $0.9 \% \mathrm{NaCl}$ containing the most bioactive hydrolases in human ALF. ${ }^{3}$ After incubation, bacilli were gently centrifuged and the supernatant containing released fragments filtersterilized for immediate use. The bacilli were washed, and suspended in RPMI containing 2.5\% human serum albumin (HSA) and $20 \mathrm{mM}$ HEPES immediately before infection. The infections were performed using M.tb counted in a Petroff Hauser chamber. The fragments in $0.9 \% \mathrm{NaCl}$ were diluted at a different MOE before use. MOE is defined as the ratio of fragments being released from a given number of $M . t b$ bacilli relative to the number of human macrophages on a monolayer, i.e., MOE 5:1 is defined as $2.0 \times 10^{5}$ macrophages exposed to fragments derived from $1.0 \times 10^{6} \mathrm{M}$.tb bacilli. For all the experiments, exposed $M . t b$ and/or their released fragments were freshly made. In all the studies, each ' $n$ ' value was obtained using fragments derived from using a different human ALF.

SDS-PAGE, monosaccharide, and fatty acid composition of released fragments. These experiments were performed as we described. ${ }^{40}$ See Supplementary Material online for details.

Isolation and preparation of human macrophages. Monocytederived macrophage monolayers for microscopy and colony-forming units (CFUs) were prepared from healthy tuberculin-negative human volunteers as previously described. ${ }^{41}$

Cytokine/chemokine levels induced by released fragments. Macrophage monolayers were exposed to released fragments at a MOE of 5:1, 10:1, 20:1, and 40:1, and TNF secretion was determined by ELISA per the manufacturer's instructions. Cytokine/chemokine/ growth-factor secretions were determined by ELISA from supernatants obtained from macrophages exposed to a MOE 20:1. For some cytokines, the levels were determined from macrophages exposed to fragments in the presence of anti-IL10-neutralizing $\mathrm{Ab}$ (at $100 \mathrm{ng} \mathrm{ml}^{-1}>95 \%$ neutralization) or isotype control (at $100 \mathrm{ng} \mathrm{ml}^{-1}$ ). For some experiments, macrophage cell lines derived from WT, TLR2-KO, and TLR4-KO mice were used to assess the dependency on TLRs for the fragments to induce production of cytokines under the same experimental conditions as described above.

Macrophage activation status and cytotoxic effects. Day 5 peripheral blood mononuclear cells (containing $\sim 10 \%$ macrophages) were exposed to released fragments and controls $(0.9 \% \mathrm{NaCl}$ or human ALF alone) at different MOEs for up to $12 \mathrm{~h}$. Surface expression of the MR, CR4, CR3, TLR2, and TLR4, and macrophage activation marker CD86 was assessed by flow cytometry gating on macrophages by size using MOE of 20:1. CD86 and HLA-DR mRNA was obtained after $3 \mathrm{~h}$ exposure to fragments and analyzed by quantitative PCR as described. ${ }^{42}$ Cell death induction by fragments alone or during infection was also determined by flow cytometry using a commercially available kit (BD Biosciences, San Jose, CA, \#559763). In all the cases $\geqslant 10,000$ cells were counted. The samples were analyzed on a BD FACSCanto II and data were analyzed using BD FACS Diva software.

Assay of ALF exposed M.tb association with macrophages in the presence or absence of released fragments. See Supplementary Material online for details.
CFU analyses of ALF component exposed M.tb in the presence or absence of released fragments. For CFU experiments, 12-day-old macrophage monolayers were washed and infected with ALF component exposed M.tb bacilli (MOI (multiplicity of infection) 1:1, triplicate wells) with or without fragments and CFUs determined as previously described. ${ }^{43}$ For some experiments, macrophages were preincubated with anti-TNF-neutralizing $\mathrm{Ab}\left(100 \mathrm{ng} \mathrm{ml}^{-1}\right)$, anti-IL-10neutralizing $\mathrm{Ab} \quad\left(100 \mathrm{ng} \mathrm{ml}^{-1}\right)$, anti-IL-6-neutralizing $\mathrm{Ab}$ (4.5 $\left.\mathrm{ng} \mathrm{ml}^{-1}\right)$, G-CSF-neutralizing $\mathrm{Ab}\left(100 \mathrm{ng} \mathrm{ml}^{-1}\right)$, or their respective isotype controls at their respective concentrations for $30 \mathrm{~min}$ and maintained throughout the course of M.tb infection. For some experiments, macrophages were pre-incubated for $30 \mathrm{~min}$ with STAT3 (at $25.75 \mu \mathrm{g} \mathrm{ml}^{-1}$ ) or NF- $\mathrm{BB}$ (at $5 \mu \mathrm{M}$ ) inhibitors, or recombinant cytokines/growth factors at half, equal, or two times the levels induced by the fragments (IL-6 (at 25, 50, and $100 \mathrm{pg} \mathrm{ml}^{-1}$ ), IL10 (at 350, 700, 1,400 $\mathrm{pg} \mathrm{ml}^{-1}$ ), and G-CSF (at 125, 250, $500 \mathrm{pg} \mathrm{ml}^{-1}$ ) and maintained throughout the course of the infection. CFUs were determined after 21 days of growth.

Phagosome-lysosome fusion of M.tb-containing phagosomes and autophagosome formation. Experiments were performed as we previously described, ${ }^{10}$ and as described in detail in the Supplementary Material online.

STAT3 and NF- KB activation induced by fragments and M.tb infection. Following $2 \mathrm{~h}$ infection, the collected samples were lysed in radioimmunoprecipitation assay buffer containing $0.25 \%$ SDS as described. ${ }^{44}$ See Supplementary Material online for details.

Oxidative response generated by macrophages exposed to fragments. The ability of fragments to activate the NADPH oxidase complex and subsequent generation of ROS; and iNOS gene expression indicative of production of NOS was assessed as described. ${ }^{45,46}$ See Supplementary Material online for details.

Statistical analysis. Prism software (GraphPad) was used to determine the statistical significance of differences in the means of experimental groups by unpaired, two-tailed Student's $t$-test. The overall data were compared by one-way analysis of variance followed by Tukey's multiple comparison tests of the means. The $P$ values of less

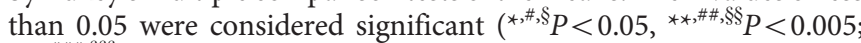
$* * *, \# \#, \$ \$ \$ P<0.0005)$.

SUPPLEMENTARY MATERIAL is linked to the online version of the paper at http://www.nature.com/mi

\section{ACKNOWLEDGMENTS}

This research was supported by NIH/NIAID [AI073856 and AI093570], and the Parker B. Francis Fellowship in pulmonary diseases to J.B.T. J.T. was partially supported by a Julie Martin Mid-Career award from The American Federation for Aging Research. JIM and JMS were partially supported by a $\mathrm{NIH} / \mathrm{NIGMS}$ T32-GM068412, and JIM also partially supported by NIH/ NIAID AI093570-S1. We thank Dr Jacob Yount for sharing the mouse WT, TLR2, and TLR4 knockout cell lines; and Dr Larry S. Schlesinger for his careful review of this manuscript. We thank the Campus Chemical Instrument Center (CCIC) and the Campus Microscopy and Imaging Facility (CMIF) at The Ohio State University (OSU) for their services. We acknowledge the facilities and programmatic support of OSU Biosafety Level 3 Program.

\section{AUTHORS CONTRIBUTIONS}

J.A., S.J.S, J.I.M., J.M.S., S.S., H.G., P.V., H.VK., G.L., L.D., S.N.S., and J.B.T. performed all the experiments presented and all participated in getting the data. J.A., S.J.S., J.I.M., and J.M.S. together with J.Z., J.T., and J.B.T. participated in the planning of all the experiments presented and data interpretation. J.B.T. also supervised the work and wrote the manuscript with the assistance of J.T., J.A., S.J.S, J.I.M., and J.M.S. J.I.M., J.M.S., and S.S. contributed equally to this work. 


\section{DISCLOSURE}

The authors declared no conflict of interest.

(c) 2017 Society for Mucosal Immunology

\section{REFERENCES}

1. WHO. WHO Tuberculosis Fact Sheet 2015. World Health Organization 15A.D. 20 March. Available from: URLhttp://www.who.int/mediacentre/ factsheets/fs104/en/.

2. Torrelles, J.B. Broadening our view about the role of Mycobacterium tuberculosis cell envelope components during infection: a battle for survival. In Understanding Tuberculosis - Analyzing the Origin of Mycobacterium tuberculosis Pathogenicity (Cardona, PJ, ed) 77-122 (Intech, Rijeka, Croatia, 2012).

3. Arcos, J., Sasindran, S.J., Fujiwara, N., Turner, J., Schlesinger, L.S. \& Torrelles, J.B. Human lung hydrolases delineate Mycobacterium tuberculosis-macrophage interactions and the capacity to control infection. J. Immunol. 187, 372-381 (2011).

4. Schlesinger, L.S. Phagocytosis and Toll-like receptors in tuberculosis. In Tuberculosis, 2nd edn (Rom, W.N. \& Garay, S.M., eds) 203-214 (Lippincott Williams \& Wilkins: New York, NY, 2004).

5. Sasindran, J. \& Torrelles, J.B. Mycobacterium tuberculosis infection and inflammation: what is beneficial for the host and for the bacterium?. Front. Microbiol. 2, 1-16 (2011).

6. Guirado, E. \& Schlesinger, L.S. Modeling the Mycobacterium tuberculosis Granuloma - the Critical Battlefield in Host Immunity and Disease. Front. Immunol. 4, 98 (2013).

7. Helke, K.L., Mankowski, J.L. \& Manabe, Y.C. Animal models of cavitation in pulmonary tuberculosis. Tuberculosis (Edinb) 86, 337-348 (2006).

8. Hawgood, S. \& Poulain, F.R. The pulmonary collectins and surfactant metabolism. Annu. Rev. Physiol. 63, 495-519 (2001).

9. Mason, R.J. Biology of alveolar type II cells. Respirology 11 (Suppl), S12-S15 (2006).

10. Kang, P.B. et al. The human macrophage mannose receptor directs Mycobacterium tuberculosis lipoarabinomannan-mediated phagosome biogenesis. J. Exp. Med. 202, 987-999 (2005).

11. Axelrod, S. etal. Delay of phagosome maturation by a mycobacterial lipid is reversed by nitric oxide. Cell Microbiol. 10, 1530-1545 (2008).

12. Kastelein, R.A., Hunter, C.A. \& Cua, D.J. Discovery and biology of IL-23 and IL-27: related but functionally distinct regulators of inflammation. Annu. Rev. Immunol. 25, 221-242 (2007).

13. O'Leary, S., O'Sullivan, M.P. \& Keane, J. IL-10 blocks phagosome maturation in Mycobacterium tuberculosis-infected human macrophages. Am. J. Respir. Cell Mol. Biol. 45, 172-180 (2010).

14. Yang, C.S., Yuk, J.M. \& Jo, E.K. The role of nitric oxide in mycobacterial infections. Immune Netw. 9, 46-52 (2009).

15. Torrelles, J.B. \& Schlesinger, L.S. Diversity in Mycobacterium tuberculosis mannosylated cell wall determinants impacts adaptation to the host. Tuberculosis (Edinb) 90, 84-93 (2010).

16. Redford, P.S., Murray, P.J. \& O'Garra, A. The role of IL-10 in immune regulation during $M$. tuberculosis infection. Mucosal Immunol. 4, 261-270 (2011).

17. Gazzinelli, R.T. et al. In the absence of endogenous IL-10, mice acutely infected with Toxoplasma gondii succumb to a lethal immune response dependent on CD4 + Tcells and accompanied by overproduction of IL-12, IFN-gamma and TNF-alpha. J. Immunol. 157, 798-805 (1996).

18. Hunter, C.A. et al. IL-10 is required to prevent immune hyperactivity during infection with Trypanosoma cruzi. J. Immunol. 158, 3311-3316 (1997).

19. Li, C., Corraliza, I. \& Langhorne, J. A defect in interleukin-10 leads to enhanced malarial disease in Plasmodium chabaudi chabaudi infection in mice. Infect. Immun. 67, 4435-4442 (1999).

20. Jung, J.Y. \& Robinson, C.M. IL-12 and IL-27 regulate the phagolysosomal pathway in mycobacteria-infected human macrophages. Cell Commun. Signal. 12, 16 (2014).

21. Liu, F.D. et al. Timed action of IL-27 protects from immunopathology while preserving defense in influenza. PLoS Pathog. 10, e1004110 (2014).

22. Mosser, D.M. The many faces of macrophage activation. J. Leukoc. Biol. 73, 209-212 (2003)
23. Retini, C., Kozel, T.R., Pietrella, D., Monari, C., Bistoni, F. \& Vecchiarelli, A. Interdependency of interleukin- 10 and interleukin-12 in regulation of T-cell differentiation and effector function of monocytes in response to stimulation with Cryptococcus neoformans. Infect. Immun. 69, 60646073 (2001).

24. Saraiva, M. \& O'Garra, A. The regulation of IL-10 production by immune cells. Nat. Rev. Immunol. 10, 170-181 (2010).

25. Day, J., Friedman, A. \& Schlesinger, L.S. Modeling the immune rheostat of macrophages in the lung in response to infection. Proc. Natl Acad. Sci. USA 106, 11246-11251 (2009).

26. Martinez, F.O. \& Gordon, S. The M1 and M2 paradigm of macrophage activation: time for reassessment. F1000Prime Rep. 6, 13 (2014).

27. Flores-Villanueva, P.O. et al. A functional promoter polymorphism in monocyte chemoattractant protein-1 is associated with increased susceptibility to pulmonary tuberculosis. J. Exp. Med. 202, 1649-1658 (2005).

28. Shanmugham, L.N. et al. Rantes potentiates human macrophage aggregation and activation responses to calcium ionophore (A23187) and activates arachidonic acid pathways. J. Biol. Regul. Homeost. Agents 20, 15-23 (2006).

29. Vesosky, B., Rottinghaus, E.K., Stromberg, P., Turner, J. \& Beamer, G. CCL5 participates in early protection against Mycobacterium tuberculosis. J. Leukoc. Biol. 87, 1153-1165 (2010).

30. Castellani, M.L. et al. Expression and secretion of RANTES (CCL5) in granulomatous calcified tissue before and after lipopolysaccharide treatment in vivo. Calcif. Tissue Int. 80, 60-67 (2007).

31. Ma, X. et al. Inhibition of IL-12 production in human monocyte-derived macrophages by TNF. J. Immunol. 164, 1722-1729 (2000).

32. Harris, J., Hope, J.C. \& Keane, J. Tumor necrosis factor blockers influence macrophage responses to Mycobacterium tuberculosis. J. Infect. Dis. 198, 1842-1850 (2008).

33. Qualls, J.E. et al. Arginine usage in mycobacteria-infected macrophages depends on autocrine-paracrine cytokine signaling. Sci. Signal. 3, ra62 (2010).

34. Via, L.E., Fratti, R.A., McFalone, M., Pagan-Ramos, E., Deretic, D. \& Deretic, $V$. Effects of cytokines on mycobacterial phagosome maturation. J. Cell Sci. 111, 897-905 (1998).

35. Cyktor, J.C. \& Turner, J. Interleukin-10 and immunity against prokaryotic and eukaryotic intracellular pathogens. Infect. Immun. 79, 2964-2973 (2011).

36. Lang, R., Rutschman, R.L., Greaves, D.R. \& Murray, P.J. Autocrine deactivation of macrophages in transgenic mice constitutively overexpressing IL-10 under control of the human CD68 promoter. J. Immunol. 168, 3402-3411 (2002).

37. Stone, K.C., Mercer, R.R., Gehr, P., Stockstill, B. \& Crapo, J.D. Allometric relationships of cell numbers and size in the mammalian lung. Am. J. Respir. Cell Mol. Biol. 6, 235-243 (1992).

38. Stone, K.C., Mercer, R.R., Freeman, B.A., Chang, L.Y. \& Crapo, J.D. Distribution of lung cell numbers and volumes between alveolar and nonalveolar tissue. Am. Rev. Respir. Dis. 146, 454-456 (1992).

39. Chroneos, Z.C., Midde, K., Sever-Chroneos, Z. \& Jagannath, C. Pulmonary surfactant and tuberculosis. Tuberculosis (Edinb) 89 (Suppl 1), S10-S14 (2009).

40. Torrelles, J.B. et al. Truncated structural variants of lipoarabinomannan in Mycobacterium leprae and an ethambutol-resistant strain of Mycobacterium tuberculosis. J. Biol. Chem. 279, 41227-41239 (2004).

41. Schlesinger, L.S. Macrophage phagocytosis of virulent but not attenuated strains of Mycobacterium tuberculosis is mediated by mannose receptors in addition to complement receptors. J. Immunol. 150, 2920-2930 (1993).

42. Arcos, J. et al. Lung mucosa lining fluid modifies Mycobacterium tuberculosis to reprogram human neutrophil killing mechanisms. J. Infect. Dis. 212, 948-958 (2015).

43. Olakanmi, O., Britigan, B.E. \& Schlesinger, L.S. Gallium disrupts iron metabolism of mycobacteria residing within human macrophages. Infect. Immun. 68, 5619-5627 (2000).

44. Xiao, Y. et al. Targeting CBLB as a potential therapeutic approach for disseminated candidiasis. Nat. Med. 22, 906-914 (2016).

45. Mohapatra, N.P. et al. Francisella acid phosphatases inactivate the NADPH oxidase in human phagocytes. J. Immunol. 184, 5141-5150 (2010).

46. Rajaram, M.V. et al. Mycobacterium tuberculosis lipomannan blocks TNF biosynthesis by regulating macrophage MAPK-activated protein kinase 2 (MK2) and microRNA miR-125b. Proc. Natl Acad. Sci. USA 108, 17408-17413 (2011). 